\title{
TWISTOR FIBRATIONS GIVING PRIMITIVE HARMONIC MAPS OF FINITE TYPE
}

\author{
RUI PACHECO
}

Received 4 April 2005 and in revised form 3 October 2005

Primitive harmonic maps of finite type from a Riemann surface $M$ into a $k$-symmetric space $G / H$ are obtained by integrating a pair of commuting Hamiltonian vector fields on certain finite-dimensional subspaces of loop algebras. We will clarify and generalize Ohnita and Udagawa's results concerning homogeneous projections $p: G / H \rightarrow G / K$, with $H \subset K$, preserving finite-type property for primitive harmonic maps.

\section{Introduction}

It is well known (cf. $[5,10]$ ) that harmonic maps from a Riemann surface $M$ to a Riemannian symmetric space $G / K$ correspond to certain holomorphic maps, the extended framings, into the loop group $\Lambda G$. An extended framing reveals intimate properties of the corresponding harmonic map.

The simplest situation occurs when the Fourier series associated to an extended framing has finitely many terms; the corresponding harmonic maps are said to have finite uniton number. All the harmonic maps in a domain of genus zero are of this kind. Again, harmonic maps obtained via twistor construction have finite uniton number. The twistortheoretic construction of harmonic maps from holomorphic data accounts for all isotropic harmonic maps from a Riemann surface into a sphere or a complex projective space. In particular, all harmonic 2-spheres in $S^{n}$ or $\mathbb{C} P^{n}$ arise in this way $[7,8,9]$.

Harmonic maps of finite type correspond to extended framings which can be obtained by integrating a pair of commuting Hamiltonian vector fields on certain finite-dimensional subspaces of loop algebras. It was shown in [4] that any nonconformal harmonic map of a 2-torus into a rank-one symmetric space $G / K$ is of finite type. Burstall [3] generalized the notion of harmonic map of finite type to the case where the target manifold admits a $k$-symmetric structure and proved that any weakly conformal nonisotropic harmonic map $\phi$ of a 2-torus into $S^{n}$ or $\mathbb{C} P^{n}$ can be lifted to a primitive harmonic map of finite type into a certain $k$-symmetric space. The $k$-symmetric spaces form a class of reductive homogeneous spaces that includes both symmetric spaces and generalized flag manifolds. 
Ohnita and Udagawa [13] showed that given a primitive harmonic map $\psi$ of finite type of $\mathbb{C}$ into a generalized flag manifold $G / H$ with its canonical $k_{1}$-symmetric structure, $\phi=p \circ \psi: \mathbb{C} \rightarrow G / K$ is also a primitive harmonic map of finite type for some choices of $K \supset H$, where $p: G / H \rightarrow G / K$ is the natural homogeneous projection over the generalized flag manifold $G / K$ with its canonical $k_{2}$-symmetric structure. For example, $\mathbb{C} P^{n}$ and the corresponding $k$-symmetric spaces used to build the primitive lifts are generalized flag manifolds and satisfy the conditions on $K$. Our main purpose in this paper is to clarify these conditions on the closed subgroup $K$ and give a generalization of Ohnita and Udagawa's result. The key observation which makes this possible is the following: what underlies their algebraic computations is the existence of an isomorphism $\Lambda_{\tau} \mathfrak{g} \rightarrow \Lambda \mathfrak{g}$, for any inner automorphism $\tau$ of order $k$ in $\mathfrak{g}$, which we can construct explicitly in several cases. Thus, clarifying this matter, we will be able to arrive at the following conclusions.

(a) The condition on the closed subgroup $K$ admits a nice geometrical formulation. $G^{\mathbb{C}}$ acts transitively on any generalized flag manifold $G / H$ with parabolic subgroups as stabilizers. A subgroup of $G^{\mathbb{C}}$ is parabolic if it can be realized as the stabilizer of some flag $V_{1} \subset V_{2} \subset \cdots \subset V_{r}=V$, for some representation $V$ of $G^{\mathbb{C}}$. Hence, associated to the generalized flag manifold $G / H$ with its canonical $k_{1}$-symmetric structure, there is a parabolic subgroup $P$. If we take another parabolic subgroup $Q$ such that $P \subset Q$, we have a new generalized flag manifold $G / K$ with its canonical $k_{2}$-symmetric structure, such that $K \supset H$. When $G^{\mathbb{C}}$ is simple, the conditions on the choice of $K$ referred to above amount to the demand that $P \subset Q$.

(b) $S^{n}$ and the corresponding $k$-symmetric spaces used to build the primitive lifts are not generalized flag manifolds. However, both symmetric structures arise in a natural way from some parabolic subgroups $P \subset Q$. Our theorem concerning homogeneous projections preserving finite-type property also holds for this kind of $k$-symmetric space. Hence, one can prove directly that any harmonic two-torus into $S^{n}$ which is covered by a primitive map of finite type is also of finite type. Ohnita and Udagawa prove this result via a totally geodesic isometric immersion into complex projective space.

\section{Primitive harmonic maps}

Let $N=G / K$ be a $k$-symmetric space with automorphism $\tau$ and associated eigenspace decomposition

$$
\mathfrak{g}^{\mathbb{C}}=\sum_{j \in \mathbb{Z}_{k}} \mathfrak{g}^{j}
$$

where $\mathfrak{g}^{j}$ is the $\omega^{j}$-eigenspace of $\tau$ and $\omega=e^{2 \pi i / k}$. We get a reductive decomposition $\mathfrak{g}=$ $\mathfrak{k} \oplus \mathfrak{m}$ by setting $\mathfrak{m}=\mathfrak{m}^{\mathbb{C}} \cap \mathfrak{g}$, where

$$
\mathfrak{m}^{\mathbb{C}}=\sum_{j \in \mathbb{Z}_{k} \backslash\{0\}} \mathfrak{g}^{j} .
$$

Let $\phi: \mathbb{C} \rightarrow N$ be a smooth map and take a lift $\psi: \mathbb{C} \rightarrow G$ with $\phi=\pi \circ \psi$, where $\pi: G \rightarrow G / K$ is the coset projection. Corresponding to the reductive decomposition is a 
decomposition of $\alpha=\psi^{-1} \mathrm{~d} \psi, \alpha=\alpha_{\mathfrak{k}}+\alpha_{\mathfrak{m}}$. Let $\alpha_{\mathfrak{m}}=\alpha_{\mathfrak{m}}^{\prime}+\alpha_{\mathfrak{m}}^{\prime \prime}$ be the type decomposition of $\alpha_{\mathfrak{m}}$ into (1,0)-form and $(0,1)$-form of $\mathbb{C}$. A map $\phi: \mathbb{C} \rightarrow N$ is said to be primitive if $\alpha_{\mathfrak{m}}^{\prime}$ is $\mathfrak{g}^{1}$-valued. If $k \geq 3$, then any primitive map $\phi: \mathbb{C} \rightarrow N$ is harmonic with respect to any naturally reductive metric on $N$ (cf. [1]). Of course, when $k=2$, all maps are primitive. Following [5], we will talk about primitive harmonic maps whenever we want to avoid treating the case of $k$-symmetric spaces with $k=2$ separately, conscious of the fact that the term "primitive" (resp., "harmonic") is redundant when $k=2$ (resp., $k>2$ ).

Suppose now that $\phi: \mathbb{C} \rightarrow N$ is a primitive harmonic map and consider the loop of 1 -forms

$$
\alpha_{\lambda}=\lambda \alpha_{\mathfrak{m}}^{\prime}+\alpha_{\mathfrak{k}}+\lambda^{-1} \alpha_{\mathfrak{m}}^{\prime \prime}
$$

Since $\alpha_{\mathfrak{m}}^{\prime}$ is $\mathfrak{g}^{1}$-valued, we may view $\alpha_{\lambda}$ as a $\Lambda \mathfrak{g}_{\tau}$-valued 1 -form, where

$$
\Lambda \mathfrak{g}_{\tau}=\left\{\xi: S^{1} \longrightarrow \mathfrak{g}(\text { smooth }): \tau(\xi(\lambda))=\xi(\omega \lambda) \text { for any } \lambda \in S^{1}\right\}
$$

Further, $\mathrm{d}+\alpha_{\lambda}$ is a loop of flat connections (cf. [5]). Conversely, suppose that $\alpha_{\lambda}$ is a loop of $\mathfrak{g}$-valued 1 -forms of the form (2.3), such that $\mathrm{d}+\alpha_{\lambda}$ is a loop of flat connections and $\alpha_{\mathfrak{m}}^{\prime}$ is $\mathfrak{g}^{1}$-valued. Then, for each $\lambda \in S^{1}$, there is a map $\psi_{\lambda}: \mathbb{C} \rightarrow G$ such that $\psi_{\lambda}^{-1} \mathrm{~d} \psi_{\lambda}=\alpha_{\lambda}$, and then $\phi_{\lambda}=\pi \circ \psi \lambda$ will be an $S^{1}$-family of primitive harmonic maps, with $\phi_{1}=\phi$ (cf. [5]). Moreover, $\psi_{\lambda}$ is unique up to left translation by a constant. We may choose these constants so that $\psi_{\lambda}\left(z_{o}\right)$ depends smoothly on $\lambda$ for some (and hence every) $z_{o} \in \mathbb{C}$. Let $\Lambda G_{\tau}$ be the infinite-dimensional Lie group corresponding to the loop Lie algebra (2.4):

$$
\Lambda G_{\tau}=\left\{\gamma: S^{1} \longrightarrow G(\text { smooth }): \gamma(\omega \lambda)=\tau(\gamma(\lambda)) \text { for all } \lambda \in S^{1}\right\}
$$

Then, we can define a smooth map $\Psi: \mathbb{C} \rightarrow \Lambda G_{\tau}$ by setting $\Psi(z)(\lambda)=\psi_{\lambda}(z) . \Psi$ is called an extended framing.

\section{Extended framings of finite type}

Let $\mathfrak{g}$ be a compact semisimple Lie algebra, $\tau: \mathfrak{g} \rightarrow \mathfrak{g}$ an automorphism of order $k$ with fixed set $\mathfrak{k}, \omega=e^{2 \pi i / k}$ the primitive $k$ th root of the unity. Let $\mathfrak{t}$ be a maximal torus of $\mathfrak{k}$. Then, $\mathfrak{t}^{\mathbb{C}}$ is a Cartan subalgebra of $\mathfrak{k}^{\mathbb{C}}$. Fix a positive root system $\Delta^{+}$. For each $X \in \mathfrak{g}$, $\operatorname{ad} X$ is skew with respect to the Killing inner product on $\mathfrak{g}$, and so has purely imaginary eigenvalues. Thus, any root $\alpha$ associated to $\mathfrak{t}^{\mathbb{C}}$ belongs to $\sqrt{-1} \mathfrak{t}^{*}$, and so $\overline{\mathfrak{k}^{\alpha}}=\mathfrak{k}^{-\alpha}$, where $\mathfrak{k}^{\alpha}$ denotes the corresponding root space. Set

$$
\mathfrak{n}=\sum_{\alpha \in \Delta^{+}} \mathfrak{k}^{\alpha}
$$

the nilpotent subalgebra given by the positive root spaces. Hence $\mathfrak{k}^{\mathbb{C}}=\mathfrak{t}^{\mathbb{C}} \oplus \mathfrak{n} \oplus \overline{\mathfrak{n}}$, where the complex conjugation is taken with respect to the real form $\mathfrak{g}$. Fixing $\mathfrak{b}=\sqrt{-1} \mathfrak{t} \oplus \overline{\mathfrak{n}}$, which is a solvable subalgebra of $\mathfrak{k}^{\mathbb{C}}, \mathfrak{k}^{\mathbb{C}}=\mathfrak{k} \oplus \mathfrak{b}$ is an Iwasawa decomposition of $\mathfrak{k}^{\mathbb{C}}$ (cf. [11]). 
Define the loop algebra

$$
\Lambda \mathfrak{g}_{\tau}^{\mathbb{C}}=\left\{\xi: S^{1} \longrightarrow \mathfrak{g}^{\mathbb{C}} \text { (smooth) }: \tau(\xi(\lambda))=\xi(\omega \lambda) \text { for any } \lambda \in S^{1}\right\} .
$$

A loop $\xi \in \Lambda \mathfrak{g}_{\tau}^{\mathbb{C}}$ has a Fourier decomposition $\xi(\lambda)=\sum_{j \in \mathbb{Z}} \xi_{j} \lambda^{j}$, with each $\xi_{j} \in \mathfrak{g}^{\mathbb{C}}$ satisfying $\tau\left(\xi_{j}\right)=\omega^{j} \xi_{j}$. Then,

$$
\Lambda \mathfrak{g}_{\tau}=\left\{\xi \in \Lambda \mathfrak{g}_{\tau}^{\mathbb{C}}: \overline{\xi_{j}}=\xi_{-j}, \xi_{j} \in \mathfrak{g}^{j}\right\}
$$

Let $d=1 \bmod k$. Define the finite-dimensional subspace $\Lambda_{d, \tau}$ of $\Lambda \mathfrak{g}_{\tau}$ by

$$
\Lambda_{d, \tau}=\left\{\xi=\sum_{j \in \mathbb{Z}} \xi_{j} \lambda^{j} \in \Lambda \mathfrak{g}_{\tau}: \xi_{j}=0 \text { for }|j|>d\right\} .
$$

For any map $\xi: \mathbb{C} \rightarrow \Lambda_{d, \tau}, \xi_{d-1}$ is $\mathfrak{k}^{\mathbb{C}}$-valued. Corresponding to decomposition $\mathfrak{k}^{\mathbb{C}}=\mathfrak{t}^{\mathbb{C}} \oplus$ $\mathfrak{n} \oplus \overline{\mathfrak{n}}$, write $\eta \in \mathfrak{k}^{\mathbb{C}}$ as $\eta=\eta_{\mathfrak{t}} \mathfrak{c}+\eta_{\mathfrak{n}}+\eta_{\overline{\mathfrak{n}}}$ and corresponding to decomposition $\mathfrak{k}^{\mathbb{C}}=\mathfrak{k} \oplus \mathfrak{b}$, write $\eta \in \mathfrak{k}^{\mathbb{C}}$ as $\eta=\eta_{\mathfrak{k}}+\eta_{\mathfrak{b}}$. It is then easy to check that $\left(\xi_{d-1} \mathrm{~d} z\right)_{\mathfrak{k}}=r\left(\xi_{d-1}\right) \mathrm{d} z+\overline{r\left(\xi_{d-1}\right)} \mathrm{d} \bar{z}$, where $r: \mathfrak{k}^{\mathbb{C}} \rightarrow \mathfrak{k}^{\mathbb{C}}$ is given by $r(\eta)=\eta_{\mathfrak{n}}+(1 / 2) \eta_{\mathfrak{t}} \mathbb{}$.

Take $\xi_{o}$ in $\Lambda_{d, \tau}$ and let $\xi: \mathbb{C} \rightarrow \Lambda_{d, \tau}$ be the unique solution of

$$
\mathrm{d} \xi=\left[\xi,\left(\lambda \xi_{d}+r\left(\xi_{d-1}\right)\right) \mathrm{d} z+\left(\lambda^{-1} \xi_{-d}+\overline{r\left(\xi_{d-1}\right)}\right) \mathrm{d} \bar{z}\right], \quad \xi(0)=\xi_{o}
$$

where $z$ is the complex coordinate on $\mathbb{C}$. For a such $\xi$,

$$
\alpha_{\lambda}=\left(\lambda \xi_{d}+r\left(\xi_{d-1}\right)\right) \mathrm{d} z+\left(\lambda^{-1} \xi_{-d}+\overline{r\left(\xi_{d-1}\right)}\right) \mathrm{d} \bar{z}
$$

is a $\Lambda_{\tau} \mathfrak{g}$-valued 1 -form on $\mathbb{C}$, since the $\lambda$-coefficient $\xi_{d}$ is $\mathfrak{g}^{1}$-valued, and $\alpha_{\lambda}$ is of the form (2.3). It happens that $\mathrm{d}+\alpha_{\lambda}$ is a loop of flat connections (cf. [5]). Thus, we can integrate to get an extended framing $\Psi: \mathbb{C} \rightarrow \Lambda G_{\tau}$ (where $G$ is a connected, compact, and semisimple Lie group with Lie algebra $\mathfrak{g}$ ), unique up to left translation by a constant loop, with $\Psi_{\lambda}^{-1} \mathrm{~d} \Psi_{\lambda}=\alpha_{\lambda}$. We call the extended framings so-obtained extended framings of finite type. A map $\xi: \mathbb{C} \rightarrow \Lambda_{d, \tau}$ which satisfies (3.5) is called a polynomial Killing field.

\section{Parabolic subalgebras}

Let $\mathfrak{g}^{\mathbb{C}}$ be a complex semisimple Lie algebra with Killing form denoted by $B$. Given a subspace $V \subset \mathfrak{g}^{\mathbb{C}}$, we will denote by $V^{\perp}$ the polar of $V$ with respect to $B$. A subalgebra $\mathfrak{p} \subset$ $\mathfrak{g}^{\mathbb{C}}$ is said to be a parabolic subalgebra if $\mathfrak{p}^{\perp}$ is a nilpotent subalgebra of $\mathfrak{g}^{\mathbb{C}}$. The relationship between such subalgebras and root systems is given in the following theorem.

Theorem 4.1 [12]. Let $\mathfrak{a}$ be a Cartan subalgebra for $\mathfrak{g}^{\mathbb{C}}, \Delta=\Delta\left(\mathfrak{g}^{\mathbb{C}} ; \mathfrak{a}\right)$ the set of roots, and $\Delta^{+}$a positive root system with simple roots $\alpha_{1}, \ldots, \alpha_{l}$. For each root $\alpha$, denote by $\mathfrak{g}^{\alpha}$ the corresponding root space. Each subset I of $\{1, \ldots, l\}$ defines a "height" function $n_{I}$ on $\Delta$ by

$$
n_{I}(\alpha)=\sum_{i \in I} n_{i}
$$


for $\alpha=\sum_{i=1}^{l} n_{i} \alpha_{i}$, and then

$$
\mathfrak{p}_{I}=\mathfrak{a} \oplus \sum_{n_{I}(\alpha) \geq 0} \mathfrak{g}^{\alpha}
$$

is a parabolic subalgebra. Moreover, every parabolic subalgebra is conjugate to a $\mathfrak{p}_{I}$ for a unique subset I of $\{1, \ldots, l\}$.

Suppose that $\mathfrak{p} \subset \mathfrak{g}^{\mathbb{C}}$ is a parabolic subalgebra. Then, $\mathfrak{p}$ makes $\mathfrak{g}^{\mathbb{C}}$ into a filtered algebra: set $\mathfrak{p}^{(0)}=\mathfrak{p}, \mathfrak{p}^{(1)}=\mathfrak{p}^{\perp}, \mathfrak{p}^{(i+1)}=\left[\mathfrak{p}^{(1)}, \mathfrak{p}^{(i)}\right]$ for $i \geq 1$, and $\mathfrak{p}^{(i)}=\mathfrak{p}^{(-i+1)^{\perp}}$ for $i<0$. The nilpotency of $\mathfrak{p}^{(1)}$ assures us of the existence of $k$ such that $\mathfrak{p}^{(k)} \neq\{0\}$ and $\mathfrak{p}^{(k+1)}=\{0\}$. Then

$$
\mathfrak{g}^{\mathbb{C}}=\mathfrak{p}^{(-k)} \supsetneq \cdots \supsetneq \mathfrak{p}^{(k)} \supsetneq \mathfrak{p}^{(k+1)}=\{0\}
$$

and for all $i$, we have $\mathfrak{p}^{(i)^{\perp}}=\mathfrak{p}^{(-i+1)}$. Call $k$ the height of $\mathfrak{p}$.

Let us consider the additional structure given by a compact real form $\mathfrak{g}$ of $\mathfrak{g}^{\mathbb{C}}$. We denote by $\xi \mapsto \bar{\xi}$ the complex conjugation on $\mathfrak{g}^{\mathbb{C}}$ with respect to the real form $\mathfrak{g}$. Set $\mathfrak{q}=\overline{\mathfrak{p}}$ (which is also a parabolic subalgebra of height $k$ ) and

$$
\mathfrak{g}_{i}=\mathfrak{p}^{(i)} \cap \mathfrak{q}^{(-i)} .
$$

The subalgebra $\mathfrak{g} \cap \mathfrak{p}$ contains a maximal torus $\mathfrak{t}$ of $\mathfrak{g}$ and $\mathfrak{t}^{\mathbb{C}}$ is a Cartan subalgebra of $\mathfrak{g}^{\mathbb{C}}$ contained in $\mathfrak{p}$. Since $\mathfrak{p}$ must have the form $\mathfrak{p}_{I}$ for some subset $I$ of simple roots with respect to $\mathfrak{t}^{\mathbb{C}}$, one can easily check that

$$
\mathfrak{g}_{r}=\sum_{n_{I}(\alpha)=r} \mathfrak{g}^{\alpha}
$$

and so

$$
\left[\mathfrak{g}_{i}, \mathfrak{g}_{j}\right] \subset \mathfrak{g}_{i+j}, \quad \mathfrak{g}^{\mathbb{C}}=\sum_{i=-k}^{k} \mathfrak{g}_{i} .
$$

Thus we are providing $\mathfrak{g}^{\mathbb{C}}$ with the structure of a graded algebra. Since $\mathfrak{g}^{\mathbb{C}}$ is semisimple (and so every derivation is an inner derivation), we conclude that there is a unique $\xi \in$ $\mathfrak{g}^{\mathbb{C}}$ with ad $\xi=i \sqrt{-1}$ on $\mathfrak{g}_{i}$ for all $i \in\{-k, \ldots, k\}$. Following [6], we call $\xi$ the canonical element of $\mathfrak{p}$ associated to the compact real form $\mathfrak{g}$. Observe that ad $\xi$ has values in $\mathfrak{g}$ when restricted to $\mathfrak{g}$. But $\mathfrak{g}$, being semisimple, has trivial center $\mathfrak{z}(\mathfrak{g})=\{0\}$, whence $\xi \in \mathfrak{g}$. At the same time, $\xi$ centralizes $\mathfrak{h}=\mathfrak{p} \cap \overline{\mathfrak{p}} \cap \mathfrak{g}$. So $\xi$ belongs to the center of $\mathfrak{h}$ in $\mathfrak{g}, \mathfrak{z}(\mathfrak{h}) \subset \mathfrak{g}$.

We will need the following two lemmas.

LEMmA 4.2. If $\mathfrak{g}^{\mathbb{C}}$ is simple and $\mathfrak{p}$ is a parabolic subalgebra with height $k$, then the center of $\mathfrak{p}^{\perp}$ is just $\mathfrak{p}^{(k)}$.

Proof. The inclusion $\mathfrak{p}^{(k)} \subset \mathfrak{z}\left(\mathfrak{p}^{\perp}\right)$ results directly from definitions. Now, since the action of $\mathfrak{h}$ on $\mathfrak{z}\left(\mathfrak{p}^{\perp}\right)$ is irreducible when $\mathfrak{g}^{\mathbb{C}}$ is simple (cf. [6, Proposition 4.3]), we must have $\mathfrak{p}^{(k)}=\mathfrak{z}\left(\mathfrak{p}^{\perp}\right)$. 
Lemma 4.3. Let $\mathfrak{p} \subset \tilde{\mathfrak{p}} \subset \mathfrak{g}^{\mathbb{C}}$ be two parabolic subalgebras with heights $k$ and $\tilde{k}$, respectively. Fix a compact real form $\mathfrak{g}$. Then, with obvious notations,

$$
\mathfrak{g}_{j} \subset \tilde{\mathfrak{g}}_{0} \oplus \tilde{\mathfrak{g}}_{1} \oplus \cdots \oplus \tilde{\mathfrak{g}}_{j}
$$

for all $j \geq 0$. Further, if $\mathfrak{g}^{\mathbb{C}}$ is simple, then

$$
\mathfrak{g}_{k-j} \subset \tilde{\mathfrak{g}}_{\tilde{k}} \oplus \tilde{\mathfrak{g}}_{\tilde{k}-1} \oplus \cdots \oplus \tilde{\mathfrak{g}}_{\tilde{k}-j}
$$

for all $j \geq 0$.

Proof. Fix a maximal torus $\mathfrak{t}$ in $\mathfrak{h} \subset \tilde{\mathfrak{h}}$ together with a positive root system $\Delta^{+}$with simple roots $\alpha_{1}, \ldots, \alpha_{l}$ so that $\mathfrak{p}=\mathfrak{p}_{I}$ and $\tilde{\mathfrak{p}}=\tilde{\mathfrak{p}}_{\tilde{I}}$. Since $\mathfrak{p} \subset \tilde{\mathfrak{p}}$, we have $\tilde{I} \subset I \subset\{1, \ldots, l\}$ and $n_{\tilde{I}}(\alpha) \leq$ $n_{I}(\alpha)$. Hence, (4.7) follows from (4.5). Suppose $\mathfrak{g}$ is simple. The adjoint representation of a simple Lie algebra is irreducible, and so $\operatorname{adg}^{\mathbb{C}}$ has a unique highest weight which is the highest root $\theta$. Observe that $n_{I}(\theta)=k$ and $n_{\widetilde{I}}(\theta)=\tilde{k}$. Since any irreducible $\mathfrak{g}^{\mathbb{C}}$-module is generated from the highest weight space by the action of vectors in the root spaces of the negatives of the simple roots, we have for any root $\alpha$,

$$
\mathfrak{g}^{\alpha}=\left[\mathfrak{g}^{-\alpha_{i_{1}}},\left[\ldots,\left[\mathfrak{g}^{-\alpha_{i_{s}}}, \mathfrak{g}^{\theta}\right] \ldots\right]\right]
$$

for suitable $i_{1}, \ldots, i_{s}$, and (4.8) follows from (4.5).

Let $G^{\mathbb{C}}$ be a connected semisimple complex Lie group with complex Lie algebra $\mathfrak{g}^{\mathbb{C}}$. A parabolic subgroup of $G^{\mathbb{C}}$ is a complex Lie subgroup which is the normalizer of a parabolic subalgebra of $\mathfrak{g}^{\mathbb{C}}$. A generalized flag manifold is a homogeneous space of the form $G^{\mathbb{C}} / P$ with $P$ a parabolic subgroup. The following facts are well known (cf. [14]): (a) all parabolic subgroups are connected and a subgroup is parabolic if and only if its algebra is parabolic; (b) if $G$ is a compact real form of $G^{\mathbb{C}}$, then $G$ acts transitively on $G^{\mathbb{C}} / P$ so that a generalized flag manifold is diffeomorphic to the real coset space $G / G \cap P$. Further, $G \cap P$ is connected and is the centralizer of a torus, while, conversely, if $H$ is the centralizer of a torus in $G$, then $H=G \cap P$ for at least one parabolic subgroup $P$ of $G^{\mathbb{C}}$.

So let $F=G^{\mathbb{C}} / P=G / H$ be a generalized flag manifold, $\mathfrak{p}$ the Lie algebra of $P, k$ the height of $\mathfrak{p}, \xi$ the canonical element of $\mathfrak{p}$ associated to the compact real form $\mathfrak{g}$ (the Lie algebra of $G$ ), and $\mathfrak{g}_{i}$ the $i \sqrt{-1}$-eigenspace of ad $\xi$. Then, the Lie algebra of $H=G \cap P$ is given by $\mathfrak{h}=\mathfrak{p} \cap \overline{\mathfrak{p}} \cap \mathfrak{g}$. Consider the inner $k+1$-automorphism $\tau: \mathfrak{g}^{\mathbb{C}} \rightarrow \mathfrak{g}^{\mathbb{C}}$ defined by

$$
\tau=\operatorname{Ad} \exp \left(\frac{2 \pi \xi}{k+1}\right)
$$

Denote by $\omega$ the primitive $(k+1)$ th root of the unity. The $\omega^{i}$-eigenspace of $\tau$ is given by $\mathfrak{g}^{i}=\mathfrak{g}_{i} \oplus \mathfrak{g}_{i-(k+1)}$, in particular $\mathfrak{g}^{0}=\mathfrak{h}^{\mathbb{C}}$. Hence, we are providing $G / H$ with the structure of an $k+1$-symmetric space, called the canonical $k+1$-symmetric structure.

\section{Twistor fibrations giving primitive harmonic maps of finite type}

The following lemma provides the key that we will use to prove the main result of this paper. 
Lemma 5.1. Let $\mathfrak{g}$ be a Lie algebra, $\tau: \mathfrak{g} \rightarrow \mathfrak{g}$ an automorphism of order $s$, and $\sigma: S^{1} \rightarrow$ Aut $\mathfrak{g}$ a group homomorphism such that $\sigma(\omega)=\tau$, where $\omega$ is the primitive sth root of the unity. Then, the map $\Gamma_{\tau}: \Lambda \mathfrak{g} \rightarrow \Lambda \mathfrak{g}_{\tau}$ given by $\Gamma_{\tau}(\gamma)(\lambda)=\sigma(\lambda) \gamma\left(\lambda^{s}\right)$ is an isomorphism.

Proof. Given $\gamma \in \Lambda \mathfrak{g}$,

$$
\begin{aligned}
\Gamma_{\tau}(\gamma)(\omega \lambda) & =\sigma(\omega \lambda) \gamma\left(\omega^{s} \lambda^{s}\right)=\sigma(\omega) \sigma(\lambda) \gamma\left(\lambda^{s}\right) \\
& =\tau\left(\sigma(\lambda) \gamma\left(\lambda^{s}\right)\right)=\tau\left(\Gamma_{\tau}(\gamma)(\lambda)\right) .
\end{aligned}
$$

Hence $\Gamma_{\tau}(\gamma) \in \Lambda \mathfrak{g}_{\tau}$. To see that $\Gamma_{\tau}$ is an isomorphism, note that $\sigma\left(\lambda^{-1 / s}\right) \gamma\left(\lambda^{1 / s}\right)$ does not depend on the choice for the sth root of $\lambda$ if $\gamma \in \Lambda \mathfrak{g}_{\tau}$. Hence we can define a map $\Gamma_{\tau}^{-1}$ : $\Lambda \mathfrak{g}_{\tau} \rightarrow \Lambda \mathfrak{g}$ by $\Gamma_{\tau}^{-1}(\gamma)(\lambda)=\sigma\left(\lambda^{-1 / s}\right) \gamma\left(\lambda^{1 / s}\right)$, for which $\Gamma_{\tau} \circ \Gamma_{\tau}^{-1}=\Gamma_{\tau}^{-1} \circ \Gamma_{\tau}=$ Id.

Suppose that

(a) $G$ is a compact Lie group with Lie algebra $\mathfrak{g}$;

(b) $\mathfrak{p} \subset \tilde{\mathfrak{p}} \subset \mathfrak{g}^{\mathbb{C}}$ are two parabolic subalgebras of $\mathfrak{g}^{\mathbb{C}}$ with heights $k>2$ and $\tilde{k} \geq 2$, respectively.

Let $\xi$ and $\tilde{\xi}$ be the canonical elements of $\mathfrak{p}$ and $\tilde{\mathfrak{p}}$, respectively. Fix $n_{1}, n_{2} \in\{0,1\}$, with $n_{1} \geq n_{2}$, and set $s=k+n_{1}$ and $\tilde{s}=\tilde{k}+n_{2}$. We can construct two automorphisms of $\mathfrak{g}^{\mathbb{C}}$ :

$$
\tau=\operatorname{Adexp}\left(\frac{2 \pi \xi}{s}\right), \quad \tilde{\tau}=\operatorname{Ad} \exp \left(\frac{2 \pi \tilde{\xi}}{\tilde{s}}\right)
$$

of order $s$ and $\tilde{s}$, respectively. Associated to $\tau$ and $\tilde{\tau}$, we have two decompositions of $\mathfrak{g}^{\mathbb{C}}$ into eigenspaces:

$$
\mathfrak{g}^{\mathbb{C}}=\sum_{i=0}^{s-1} \mathfrak{g}^{i}=\sum_{i=0}^{\tilde{s}-1} \tilde{\mathfrak{g}}^{i},
$$

where

$$
\mathfrak{g}^{i}=\sum_{j=i \bmod s} \mathfrak{g}_{j}, \quad \tilde{\mathfrak{g}}^{i}=\sum_{j=i \bmod \tilde{s}} \tilde{\mathfrak{g}}_{j}
$$

Now suppose that

(c) $\mathfrak{g}^{\mathbb{C}}$ is simple.

In this case, Lemma 4.3 gives $\mathfrak{g}_{-k} \subset \tilde{\mathfrak{g}}_{-\widetilde{k}}$ and $\mathfrak{g}_{k} \subset \tilde{\mathfrak{g}}_{\tilde{k}}$; on the other hand, $\mathfrak{g}_{0}=\mathfrak{p} \cap \overline{\mathfrak{p}} \subset$ $\tilde{\mathfrak{p}} \cap \overline{\widetilde{\mathfrak{p}}}=\tilde{\mathfrak{g}}_{0}$. Hence $\mathfrak{g}^{0} \subset \tilde{\mathfrak{g}}^{0}$. Further, since $\mathfrak{g}^{0}$ and $\tilde{\mathfrak{g}}^{0}$ are closed under conjugation, there are subgroups $K$ and $\widetilde{K}$ of $G$, with Lie algebras $\mathfrak{k}$ and $\tilde{\mathfrak{k}}$, such that $K \subset \widetilde{K}, \mathfrak{k}^{\mathbb{C}}=\mathfrak{g}^{0}$, and $\widetilde{\mathfrak{k}} \mathbb{C}=\tilde{\mathfrak{g}}^{0}$. In fact, we are providing $G / K$ and $G / \widetilde{K}$ with structures of $s$ - and $\tilde{s}$-symmetric space, respectively. We observe that when $n_{1}=n_{2}=1, G / K$ and $G / \widetilde{K}$ are generalized flag manifolds. With these definitions, we have the following theorem.

Theorem 5.2. Assume (a), (b), and (c) above. Then if $\psi: \mathbb{C} \rightarrow G / K$ is a primitive map of finite type, so is $p \circ \psi: \mathbb{C} \rightarrow G / \widetilde{K}$, where $p: G / K \rightarrow G / \widetilde{K}$ is the canonical homogeneous projection. 
Proof. Fix a maximal torus $\mathfrak{t}$ of $\mathfrak{g}$ which is contained in $\mathfrak{k} \subset \widetilde{\mathfrak{k}}$. Denote by $\Delta$ the set of roots in $\mathfrak{g}^{\mathbb{C}}$ associated to the Cartan subalgebra $\mathfrak{t}^{\mathbb{C}}$ and define the subset $S \subset \Delta$ by $\alpha \in S$ if and only if $\mathfrak{g}^{\alpha} \subset \mathfrak{p}^{\perp} \cap \tilde{\mathfrak{g}}_{0}$ or $\mathfrak{g}^{\alpha} \subset \overline{\mathfrak{p}}^{\perp} \cap \tilde{\mathfrak{g}}_{-\widetilde{s}} . S$ is nonempty and satisfies (i) $S \cap-S=\varnothing$; (ii) $S$ is closed. Any subset of $\Delta$ satisfying these conditions can be extended to a positive root system (cf. [2]). Let $\Delta^{+}$be such extension of $S$ and let $\mathfrak{n}$ be the subalgebra generated by all the positive root spaces. According to our choices, we observe that

$$
\begin{aligned}
& \mathfrak{g}^{1} \cap \tilde{\mathfrak{g}}^{0} \cap \overline{\mathfrak{n}}=\{0\}, \\
& \mathfrak{g}^{1} \cap \tilde{\mathfrak{g}}^{2}=\mathfrak{g}^{1} \cap \tilde{\mathfrak{g}}^{3}=\cdots=\mathfrak{g}^{1} \cap \tilde{\mathfrak{g}}^{\widetilde{s}-1}=\{0\} .
\end{aligned}
$$

In fact, since

$$
\begin{gathered}
\mathfrak{g}^{1} \cap \tilde{\mathfrak{g}}^{0} \cap \overline{\mathfrak{n}}=\left(\mathfrak{g}_{1-s} \cap \tilde{\mathfrak{g}}_{0}\right) \oplus\left(\mathfrak{g}_{1} \cap \tilde{\mathfrak{g}}_{\tilde{s}}\right), \\
\mathfrak{g}^{1} \cap \tilde{\mathfrak{g}}^{j}=\left(\mathfrak{g}_{1} \oplus \mathfrak{g}_{1-s}\right) \cap\left(\tilde{\mathfrak{g}}_{j} \oplus \tilde{\mathfrak{g}}_{j-\widetilde{s}}\right)=\left(\mathfrak{g}_{1} \cap \tilde{\mathfrak{g}}_{j}\right) \oplus\left(\mathfrak{g}_{1-s} \cap \tilde{\mathfrak{g}}_{j-\widetilde{s}}\right),
\end{gathered}
$$

equalities (5.5) follow from (4.7) and (4.8).

Starting with the canonical elements $\xi$ and $\tilde{\xi}$ of $\mathfrak{p}$ and $\tilde{\mathfrak{p}}$, respectively, we can define two loops of automorphisms $\sigma, \tilde{\sigma}: S^{1} \rightarrow$ Aut $\mathfrak{g}$ by

$$
\sigma\left(\lambda=e^{i \theta}\right)=\operatorname{Ad} \exp (\theta \xi), \quad \tilde{\sigma}\left(\lambda=e^{i \theta}\right)=\operatorname{Ad} \exp (\theta \tilde{\xi}) .
$$

Note that $\sigma(\omega)=\tau$ and $\tilde{\sigma}(\tilde{\omega})=\tilde{\tau}$. We also have $\sigma(\lambda) \mathfrak{g}_{j}=\operatorname{Ad} \exp (\theta \xi) \mathfrak{g}_{j}=\lambda^{j} \mathfrak{g}_{j}$, and in the same way $\tilde{\sigma}(\lambda) \tilde{\mathfrak{g}}_{j}=\operatorname{Ad} \exp (\theta \tilde{\xi}) \tilde{\mathfrak{g}}_{j}=\lambda^{j} \tilde{\mathfrak{g}}_{j}$. By Lemma 5.1, we have an isomorphism $\Gamma: \Lambda_{\tau} \mathfrak{g} \rightarrow \Lambda_{\tilde{\tau}} \mathfrak{g}$ defined by

$$
\Gamma(\eta)(\lambda)=\tilde{\sigma}(\lambda) \sigma\left(\lambda^{-\tilde{s} / s}\right) \eta\left(\lambda^{\tilde{s} / s}\right)
$$

We will also denote by $\Gamma: \Lambda_{\tau} G \rightarrow \Lambda_{\tau} G$ the corresponding isomorphism between loop Lie groups. Set $\nu(\lambda)=\tilde{\sigma}(\lambda) \sigma\left(\lambda^{-\tilde{s} / s}\right)$. Hence,

$$
\nu(\lambda)=\left\{\begin{array}{lll}
\lambda^{j-i(\tilde{s} / s)} & \text { on } \mathfrak{g}^{i} \cap \tilde{\mathfrak{g}}^{j}, & \text { for } i, j \neq 0, \\
\lambda^{-i(\widetilde{s} / s)} & \text { on } \mathfrak{g}^{i} \cap \tilde{\mathfrak{g}}^{0} \cap \mathfrak{n}, & \text { for } i \neq 0, \\
\lambda^{\tilde{s}-i(\widetilde{s} / s)} & \text { on } \mathfrak{g}^{i} \cap \tilde{\mathfrak{g}}^{0} \cap \overline{\mathfrak{n}}, & \text { for } i \neq 0, \\
\mathrm{Id} & \text { on } \mathfrak{g}^{0} \cap \tilde{\mathfrak{g}}^{0} . &
\end{array}\right.
$$

Let now $\Psi: \mathbb{C} \rightarrow \Lambda_{\tau} G$ be an extended framing of $\psi$ with associated Killing field $\eta: \mathbb{C} \rightarrow$ $\Lambda_{d, \tau}, \eta=\sum_{j \leq d} \eta_{j} \lambda^{j}$, where $d=1 \bmod s$, for the fixed Cartan subalgebra $\mathfrak{t}^{\mathbb{C}}$ and positive root system $\Delta^{+}$. For $1 \leq i \leq s-1$ and $n \in \mathbb{N}$, we have

$$
\nu(\lambda) \eta_{I} \lambda^{I(\tilde{s} / s)}=\sum_{j=1}^{\tilde{s}-1} \lambda^{j+n \tilde{s}}\left(\eta_{I}\right)_{\mathfrak{g}^{i} \cap \tilde{\mathfrak{g}}^{j}}+\lambda^{n \widetilde{s}}\left(\eta_{I}\right)_{\mathfrak{g}^{i} \cap \tilde{\mathfrak{g}}^{0} \cap \mathfrak{n}}+\lambda^{\widetilde{s}(1+n)}\left(\eta_{I}\right)_{\mathfrak{g}^{i} \cap \tilde{\mathfrak{g}}^{0} \cap \overline{\mathfrak{n}}}
$$

where $I=i+n s$. At the same time,

$$
\nu(\lambda) \eta_{n s} \lambda^{(n s)(\tilde{s} / s)}=\eta_{n s} \lambda^{n \tilde{s}} .
$$


From (5.5), (5.10), and (5.11), we conclude that the top terms of $\tilde{\eta}(\lambda)=\Gamma(\eta)(\lambda)$ are

$$
\begin{gathered}
\lambda^{\tilde{s} N+1} \tilde{\eta}_{\tilde{s} N+1}=\lambda^{\tilde{s} N+1}\left(\eta_{d}\right)_{\mathfrak{g}^{1} \cap \tilde{\mathfrak{g}}^{1},} \\
\lambda^{\tilde{s} N} \tilde{\eta}_{\tilde{s} N}=\lambda^{\tilde{s} N}\left\{\eta_{d-1}+\left(\eta_{d}\right)_{\mathfrak{g}^{1} \cap \tilde{\mathfrak{g}}^{0} \cap \mathfrak{n}}+\sum_{i=1}^{s-1}\left(\eta_{i+(N-1) s}\right)_{\mathfrak{g}^{i} \cap \tilde{\mathfrak{g}}^{0} \cap \overline{\mathfrak{n}}}\right\},
\end{gathered}
$$

where $N \in \mathbb{N}$ is defined by $d=1+N$ s. So $\tilde{\eta} \in \Lambda_{\tilde{d}, \tilde{\tau}}$, with $\tilde{d}=(\tilde{s} / s)(d-1)+1$.

Now, defining $\tilde{\Psi}=\Gamma(\Psi)$, we have

$$
\begin{aligned}
\tilde{\Psi}^{-1} \tilde{\Psi}_{z} & =v(\lambda)\left(\Psi^{-1}\left(\lambda^{\tilde{s} / s}\right) \Psi_{z}\left(\lambda^{\tilde{s} / s}\right)\right) \\
& =v(\lambda)\left(\lambda^{\tilde{s} / s} \eta_{d}+r\left(\eta_{d-1}\right)\right) \\
& =\lambda\left(\eta_{d}\right)_{\mathfrak{g}^{1} \cap \tilde{\mathfrak{g}}^{1}}+\left(\eta_{d}\right)_{\mathfrak{g}^{1} \cap \tilde{\mathfrak{g}}^{0} \cap \mathfrak{n}}+r\left(\eta_{d-1}\right) \\
& =\lambda \tilde{\eta}_{\tilde{d}}+\tilde{r}\left(\tilde{\eta}_{\tilde{d}_{-1}}\right) .
\end{aligned}
$$

Then, we see that $p \circ \psi$ is also of finite type.

Example 5.3. Fix in $\mathbb{C}^{n}$ the usual Hermitian inner product. Let $I=\left\{i_{1}<\cdots<i_{r}=n\right\} \subset$ $\{1, \ldots, n\}$ be a multi-index. A flag of index $I$ is a filtration of $\mathbb{C}^{n}$ by subspaces $V_{i}, V_{1} \subset$ $\cdots \subset V_{r}=\mathbb{C}^{n}$, with $\operatorname{dim} V_{j}=i_{j}$. Then, we find that

$$
\mathfrak{p}=\left\{T \in \mathfrak{s l}(n, \mathbb{C}): T V_{j} \subset V_{j} \forall j\right\}
$$

is a parabolic subalgebra of $\mathfrak{s l}(n, \mathbb{C})=\mathfrak{s u}^{\mathbb{C}}(n)$ for which

$$
\mathfrak{p}^{(i)}=\left\{T \in \mathfrak{s l}(n, \mathbb{C}): T V_{j} \subset V_{j-i} \forall j\right\}
$$

where we set $V_{j}=\{0\}$ for $j \leq 0 . \mathfrak{p}$ has height $r-1$. We may define mutually orthogonal subspaces $E_{1}, \ldots, E_{r}$ by $E_{i}=V_{i} \cap V_{i-1}^{\perp}$. Then

$$
\mathfrak{p} \cap \mathfrak{s u}(n)=\left\{T \in \mathfrak{s u}(n): T E_{j} \subset E_{j} \forall j\right\} \cong \mathfrak{s}\left(\mathfrak{u}\left(i_{1}\right) \times \cdots \times \mathfrak{u}\left(n-i_{r-1}\right)\right) .
$$

The corresponding generalized flag manifold is therefore

$$
F_{I}=\frac{S U(n)}{S\left(U\left(i_{1}\right) \times \cdots \times U\left(n-i_{r-1}\right)\right)} .
$$

Consider a new flag of index $J=\left\{j_{1}<\cdots<j_{s}=n\right\} \subset\{1, \ldots, n\}, W_{1} \subset W_{2} \subset \cdots \subset W_{s}=$ $\mathbb{C}^{n}$, with $s<r$. Suppose that for each $j \in\{1, \ldots, s\}$, there is some $i \geq j$, with $i \in\{1, \ldots, r\}$, such that $W_{j}=V_{i}$. Then we find a new parabolic subalgebra of $\mathfrak{s l}(n, \mathbb{C})$,

$$
\tilde{\mathfrak{p}}=\left\{T \in \mathfrak{s l}(n, \mathbb{C}): T W_{j} \subset W_{j} \forall j\right\}
$$

with height $s-1$, for which $\mathfrak{p} \subset \tilde{\mathfrak{p}}$. The corresponding generalized flag manifold is now $F_{J}$ and by Theorem 5.2, we conclude that a primitive harmonic map of finite type into $F_{I}$ gives rise by projection to a primitive harmonic map of finite type into $F_{J}$. 
Remark 5.4. Burstall proves in [3] that any weakly conformal nonisotropic harmonic map from the 2-torus to $\mathbb{C} P^{n-1}=S U(n) / S(U(1) \times U(n-1))$ is covered by a primitive map of finite type into a certain generalized flag manifold $F_{I}$, with $I=\left\{i_{1}<\cdots<i_{r}=n\right\}$ such that $i_{1}=1$. On the other hand, in [4] the authors proved that any nonconformal harmonic map of a 2-torus into a rank-one symmetric space $G / K$ is of finite type. Combining these results with Theorem 5.2, we conclude with Ohnita and Udagawa that any nonisotropic harmonic map from the 2-torus to $\mathbb{C} P^{n-1}$ is of finite type.

Theorem 5.5. Suppose that $G$ is a compact semisimple Lie group. Let $G / K$ and $G / \widetilde{K}$ be two generalized flag manifolds associated to parabolic subalgebras $\mathfrak{p}$ and $\tilde{\mathfrak{p}}$ such that $\mathfrak{p} \subset \tilde{\mathfrak{p}}$ and $\mathfrak{p}^{(k)} \subset \widetilde{\mathfrak{p}}^{(\tilde{k})}$, where $k$ and $\tilde{k}$ are the heights of $\mathfrak{p}$ and $\tilde{\mathfrak{p}}$, respectively. Then if $\psi: \mathbb{C} \rightarrow G / K$ is a primitive map of finite type, so is $p \circ \psi: \mathbb{C} \rightarrow G / \widetilde{K}$, where $p: G / K \rightarrow G / \widetilde{K}$ is the canonical projection.

Proof. We observe that the condition " $\mathfrak{g} \mathbb{C}$ is simple" in Lemma 4.3 could be replaced by the condition $\mathfrak{p}^{(k)} \subset \widetilde{\mathfrak{p}}^{(\tilde{k})}$ in order to ensure (4.8). Hence, the proof of Theorem 5.2 can also be applied in this setting.

Remark 5.6. Let $G$ be a semisimple Lie group and let $G / K$ be a generalized flag manifold with the canonical $k+1$-symmetric structure $\tau$ and $\mathfrak{p}$ the corresponding parabolic subalgebra. Pick a Cartan subalgebra and a positive root system $\Delta^{+}$so that $\mathfrak{p}=\mathfrak{q}_{I}$ for some subset $I$. Let $\mathfrak{n}$ be the subalgebra generated by the positive root spaces. Suppose that $\widetilde{K}$ is a closed subgroup of $G$ satisfying that (i) $K \subset \widetilde{K}$ and $G / \tilde{K}$ is a generalized flag manifold with the canonical $\tilde{k}+1$-symmetric structure $\tilde{\tau}$ for some $2 \leq \tilde{k}+1<k+1$; (ii) the canonical decomposition $\mathfrak{g}=\tilde{\mathfrak{k}} \oplus \tilde{\mathfrak{m}}$ is $\tau$-stable and orthogonal; (iii) the eigenspace decomposition of $\mathfrak{g}^{\mathbb{C}}$ with respect to $\tilde{\tau}, \mathfrak{g}^{\mathbb{C}}=\sum \tilde{\mathfrak{g}}^{j}$, with $\tilde{\mathfrak{g}}^{0}=\tilde{\mathfrak{k}}^{\mathbb{C}}$, satisfies $\mathfrak{g}^{1} \cap \overline{\mathfrak{n}} \subset \tilde{\mathfrak{g}}^{1}$ and $\mathfrak{g}^{j} \cap \tilde{\mathfrak{g}}^{s}=0$ for $j=1, \ldots, \widetilde{k}-1$ and $s=j+1, \ldots, \widetilde{k}$. In [13, Theorem 3.5], Ohnita and Udagawa proved that under these conditions, the homogeneous projection $p: G / K \rightarrow G / \widetilde{K}$ transforms primitive maps of finite type in primitive maps of finite type. Consider the parabolic subalgebra defined by

$$
\tilde{\mathfrak{p}}=\tilde{\mathfrak{g}}^{0} \oplus \sum_{j \geq 1} \tilde{\mathfrak{g}}^{j} \cap \mathfrak{n}
$$

We observe that the generalized flag manifold $G / \widetilde{K}$ is associated to $\tilde{\mathfrak{p}}$ and that these conditions on $\widetilde{K}$ imply that $\mathfrak{p} \subset \widetilde{\mathfrak{p}}$ and $\mathfrak{p}^{(k)} \subset \widetilde{\mathfrak{p}}^{(\tilde{k})}$. Hence, Ohnita and Udagawa's theorem is a particular case of Theorem 5.5 .

Example 5.7. Let $V=\mathbb{R}^{2 n+1},(\cdot, \cdot)$ the usual inner product in $V$, and $(\cdot, \cdot)^{\mathbb{C}}$ its complex bilinear extension. Fix $r \in \mathbb{N}$ with $r<n+1$ and let $F^{r}\left(S^{2 n}\right)$ be the bundle of isotropic flags over $S^{2 n}$ with fiber

$$
F_{x}^{r}\left(S^{2 n}\right)=\left\{w_{1} \subset \cdots \subset w_{r} \subset T_{x}^{\mathbb{C}} S^{2 n}: \text { each } w_{j} \text { is an isotropic } j \text {-plane }\right\}
$$

Here, isotropy is with respect to the complexified metric on $T^{\mathbb{C}} S^{2 n}$. One can easily check 
that $G=S O(2 n+1)$ acts transitively on $F^{r}\left(S^{2 n}\right)$ with stabilizers conjugate to

$$
\overbrace{S O(2) \times \cdots \times S O(2)}^{r \text { times }} \times S O(2 n-2 r) \text {. }
$$

Fix a base point $\left(m, w_{1} \subset \cdots \subset w_{r}\right) \in F^{r}\left(S^{2 n}\right)$ with stabilizer $H$ and let $\ell_{0}=\operatorname{span}_{\mathbb{R}}\{m\}$. Orthogonalize to obtain isotropic lines $\ell_{1}, \ldots, \ell_{r}$ and a real subspace $\ell_{r+1}$ in $T_{m}^{\mathbb{C}} S^{2 n}$ so that

$$
V^{\mathbb{C}}=\ell_{0}^{\mathbb{C}} \oplus \sum_{i=1}^{r}\left(\ell_{i} \oplus \overline{\ell_{i}}\right) \oplus \ell_{r+1}, \quad w_{j}=\sum_{i=1}^{j} \ell_{i}
$$

are orthogonal decompositions. Take $k=2 r+2$. Let $\omega$ be the usual $k$ th root of unity and define $Q \in O(2 n+1)$ by $Q=\omega^{j}$ on $\ell_{j}$. Let $\tau$ be the order $k$ automorphism of $S O(2 n+1)$ given by conjugation by $Q$. The identity component of the fixed set of $\tau$ is precisely the stabilizer $H$, so that $F^{r}\left(S^{2 n}\right)$ is a $k$-symmetric space. Consider the usual isomorphism $\mathfrak{s o}(2 n+1) \cong \wedge^{2} \mathbb{R}^{2 n+1}$ :

$$
(a \wedge b)(x)=(a, x) b-(b, x) a .
$$

The adjoint action of $S O(2 n+1)$ on $\wedge^{2} \mathbb{R}^{2 n+1}$ is given by

$$
\operatorname{Ad}_{g}(a \wedge b)=g(a) \wedge g(b)
$$

The associate reductive decomposition $\mathfrak{g}=\mathfrak{h} \oplus \mathfrak{m}$ is given by

$$
\begin{gathered}
\mathfrak{h}^{\mathbb{C}}=\sum_{i=1}^{r}\left(\overline{\ell_{i}} \wedge \ell_{i}\right) \oplus \wedge^{2} \ell_{r+1}, \\
\mathfrak{m}^{\mathbb{C}}=\sum_{0 \leq i<j \leq r+1}\left(\ell_{i} \wedge \ell_{j}\right) \oplus \sum_{0 \leq i \neq j \leq r+1}\left(\overline{\ell_{i}} \wedge \ell_{j}\right) \oplus \sum_{0 \leq i<j \leq r+1}\left(\overline{\ell_{i}} \wedge \overline{\ell_{j}}\right) .
\end{gathered}
$$

Moreover, the $\omega^{j}$-eigenspace of $\tau$ is

$$
\mathfrak{g}^{j}=\ell_{0}^{\mathbb{C}} \wedge \ell_{j} \oplus \sum_{i=1}^{r-j+1}\left(\overline{\ell_{i}} \wedge \ell_{j+i}\right) \oplus \sum_{i+s=j ; i<s}\left(\ell_{i} \wedge \ell_{s}\right) \oplus \sum_{i+s=j \bmod k ; 1 \leq i<s}\left(\overline{\ell_{i}} \wedge \overline{\ell_{s}}\right),
$$

for $j \in\{1, \ldots, r\}$, and

$$
\mathfrak{g}^{r+1}=\ell_{0}^{\mathbb{C}} \wedge \ell_{r+1} \oplus \sum_{i+s=r+1 ; i<s}\left(\ell_{i} \wedge \ell_{s}\right) \oplus \sum_{(i+s)=r+1 ; i<s}\left(\overline{\ell_{i}} \wedge \overline{\ell_{s}}\right) .
$$

For $j \in\{r+2, \ldots, 2 r+1\}$, we have $\mathfrak{g}^{j}=\overline{\mathfrak{g}^{2 r+2-j}}$. Let $W$ be a maximal isotropic subspace of $\ell_{r+1}$. So $\ell_{r+1}=W \oplus \bar{W}$.

Define $Q_{S} \in O(2 n+1)$ by $Q_{S}=-1$ on $\ell_{j}$, for every $\ell_{j} \in\{1, \ldots, r+1\}$, and $Q_{S}=1$ on $\ell_{0}^{\mathbb{C}}$. Let $\tilde{\tau}$ be the order- 2 automorphism of $S O(2 n+1)$ given by conjugation by $Q_{S}$. The identity component of the fixed set of $\tilde{\tau}$ is precisely the stabilizer $K$ of $m$. Hence, $S^{2 n}$ is a 
symmetric space. The associated reductive decomposition $\mathfrak{g}=\mathfrak{k} \oplus \mathfrak{q}$ is given by $\mathfrak{k}^{\mathbb{C}}=\wedge^{2} \tilde{V}$ and $\mathfrak{q}^{\mathbb{C}}=\ell_{0}^{\mathbb{C}} \wedge \tilde{V}$, where $\tilde{V}=\sum_{i=1}^{r}\left(\ell_{i} \oplus \overline{\ell_{i}}\right) \oplus \ell_{r+1}$.

We now describe the structure of $\mathfrak{s o}(2 n+1, \mathbb{C})$. Fix a Hermitian basis for $V^{\mathbb{C}}$,

$$
v_{0}, v_{1}, \ldots, v_{r}, v_{r+1}, \ldots, v_{n}, v_{n+1}, \ldots, v_{2 n}
$$

such that, $\overline{v_{i}}=v_{i+n}$ for each $i \in\{1, \ldots, n\}, \ell_{i}=\operatorname{span}_{\mathbb{C}}\left\{v_{i}\right\}$ for each $i \in\{0, \ldots, r\}$, and $W$ is generated by $\left\{v_{r+1}, \ldots, v_{n}\right\}$. The subalgebra $\mathfrak{t}^{\mathbb{C}}$ generated by the vectors $H_{i}=\overline{v_{i}} \wedge v_{i}$ with $1 \leq i \leq n$ is a Cartan subalgebra of $\mathfrak{s o}(2 n+1, \mathbb{C})$. In the dual space $\left(\mathfrak{t}^{\mathbb{C}}\right)^{*}$, consider the dual basis $\left\{L_{i}\right\}: L_{i}\left(H_{j}\right)=\delta_{i j}$. The subset of roots

$$
\Delta^{+}=\left\{L_{i}+L_{j}\right\}_{i<j} \cup\left\{L_{j}-L_{i}\right\}_{i<j} \cup\left\{L_{i}\right\}_{1 \leq i \leq n}
$$

forms a positive set of roots. Associated to $L_{i}+L_{j}$, we have the root space $\left\langle v_{i} \wedge v_{j}\right\rangle, i<j$; associated to $L_{j}-L_{i}$, we have the root space $\left\langle\overline{v_{i}} \wedge v_{j}\right\rangle, i<j$; associated to $L_{i}$, we have the root space $\left\langle v_{i} \wedge v_{0}\right\rangle, 1 \leq i \leq n$. Set $\alpha_{0}=L_{1}$ and $\alpha_{i}=L_{i+1}-L_{i}$ for $i \in\{1, \ldots, n-1\}$. So $\alpha_{0}, \ldots, \alpha_{n-1}$ is the set of simple roots associated with $\Delta^{+}$.

Define the subset $I \subset\{0, \ldots, n-1\}$ by $i \in I$ if $\mathfrak{g}^{\alpha_{i}} \subset \mathfrak{m}^{\mathbb{C}}$. Note that

(i) $L_{j}=\alpha_{0}+\alpha_{1}+\cdots+\alpha_{j-1}$ if $1 \leq j \leq n$. So $n_{I}\left(L_{j}\right)=j$ for $1 \leq j \leq r$, and $n_{I}\left(L_{j}\right)=$ $r+1$ for $j>r$

(ii) $L_{i+j}-L_{i}=\alpha_{i}+\cdots+\alpha_{i+j-1}$. So $n_{I}\left(L_{i+j}-L_{i}\right)=j$ for $i+j \leq r+1, n_{I}\left(L_{i+j}-L_{i}\right)=$ $r+1-i$ for $i+j>r+1, i<r+1$, and $n_{I}\left(L_{i+j}-L_{i}\right)=0$ for $i \geq r+1$;

(iii) $n_{I}\left(L_{i}+L_{j}\right)=n_{I}\left(L_{i}\right)+n_{I}\left(L_{j}\right)$.

Clearly, $\max _{\alpha \in \Delta^{+}}\left\{n_{I}(\alpha)\right\}=k$. Consider then the height- $k$ parabolic subalgebra $\mathfrak{p}=\mathfrak{p}_{I}$. Let $\xi$ be the canonical element of $\mathfrak{p}$ and $\mathfrak{g}_{j}$ the $\sqrt{-1} j$-eigenspace of ad $\xi$. Then,

$$
\begin{gathered}
\mathfrak{g}_{0}=W \wedge \bar{W} \oplus \sum_{i=1}^{r}\left(\overline{\ell_{i}} \wedge \ell_{i}\right), \\
\mathfrak{g}_{r+1}=\ell_{0}^{\mathbb{C}} \wedge W \oplus \sum_{i+s=r+1 ; i<s}\left(\ell_{i} \wedge \ell_{s}\right), \\
\mathfrak{g}_{2 r+2}=W \wedge W
\end{gathered}
$$

and for $0<j<r+1$, we have

$$
\begin{aligned}
\mathfrak{g}_{j} & =\ell_{0}^{\mathbb{C}} \wedge \ell_{j} \oplus \sum_{i=1}^{r-j}\left(\overline{\ell_{i}} \wedge \ell_{i+j}\right) \oplus \overline{\ell_{r-j+1}} \wedge W \oplus \sum_{i+s=j ; i<s}\left(\ell_{i} \wedge \ell_{s}\right), \\
\mathfrak{g}_{r+1+j} & =\sum_{i, s \neq r+1 ; i+s=r+1+j ; i<s}\left(\ell_{i} \wedge \ell_{s}\right) \oplus \ell_{j} \wedge W .
\end{aligned}
$$

So it is now easy to check that $\tau=\operatorname{Ad} \exp (2 \pi \xi / k)$.

Define the new subset $J \subset\{0, \ldots, n-1\}$ by $i \in J$ if $\mathfrak{g}^{\alpha_{i}} \subset \mathfrak{q}^{\mathbb{C}}$. Note that (i) $n_{J}\left(L_{j}\right)=1$; (ii) $n_{J}\left(L_{i+j}-L_{i}\right)=0$; (iii) $n_{J}\left(L_{i}+L_{j}\right)=n_{J}\left(L_{i}\right)+n_{J}\left(L_{j}\right)=2$. So $\max _{\alpha \in \Delta^{+}}\left\{n_{J}(\alpha)\right\}=2$. Consider then the height-2 parabolic subalgebra $\tilde{\mathfrak{p}}=\mathfrak{p}_{J}$. Clearly, $\mathfrak{p} \subset \tilde{\mathfrak{p}}$. Let $\tilde{\xi}$ be the canonical 
element of $\tilde{\mathfrak{p}}$ and $\tilde{\mathfrak{g}}_{j}$ the $\sqrt{-1} j$-eigenspace of ad $\tilde{\xi}$. Then,

$$
\begin{gathered}
\mathfrak{g}_{0}=\sum_{1 \leq i \neq j \leq r}\left(\overline{\ell_{i}} \wedge \ell_{j}\right) \oplus \sum_{i=1}^{r}\left(\overline{\ell_{i}} \wedge W\right) \oplus \bar{W} \wedge W, \\
\mathfrak{g}_{1}=\sum_{j=1}^{r}\left(\ell_{0}^{\mathbb{C}} \wedge \ell_{j}\right) \oplus\left(\ell_{0}^{\mathbb{C}} \wedge W\right), \\
\mathfrak{g}_{2}=\sum_{1 \leq i<j \leq r}\left(\ell_{i} \wedge \ell_{j}\right) \oplus(W \wedge W) .
\end{gathered}
$$

One can easily check that $\tilde{\tau}=\operatorname{Ad} \exp (\pi \tilde{\xi})$. Thus, $F^{r}\left(S^{2 n}\right)$ and $S^{2 n}$ satisfy the conditions of Theorem 5.2.

Remark 5.8. Burstall proves in [3] that any weakly conformal nonisotropic harmonic map from the 2-torus to $S^{n}$ can be lifted to a primitive map of finite type into a certain generalized flag manifold of the form $F^{r}\left(S^{n}\right)$. On the other hand, in [4] the authors proved that any nonconformal harmonic map of a 2-torus into a rank-one symmetric space $G / K$ is of finite type. Combining these results with Theorem 5.2, we conclude that any nonisotropic harmonic map of 2-torus into a sphere $S^{n}$ is of finite type (when $n$ is odd, we can view $S^{n}$ as an equator of $\left.S^{n+1}\right)$. In [13], Ohnita and Udagawa prove this same result by constructing an embedding of $\mathfrak{s o}(n+1)$ into $\mathfrak{s u}(n+1)$ such that for each $j$, the $\mathfrak{g}^{j}$-subspace for the $k$-symmetric structure of $F^{r}\left(S^{n}\right)$ is mapped into the $\mathfrak{g}^{j}$-subspace for the canonical $k$-symmetric structure on the generalized flag manifold over $\mathbb{C} P^{n}$. Our treatment of the sphere case is more direct and arises in a general setting.

Example 5.9. Let $\mathfrak{p} \subset \mathfrak{g}^{\mathbb{C}}$ be a parabolic subalgebra of height $k$. Let $F=G / H$ be the corresponding generalized flag manifold with canonical $k+1$-symmetric structure denoted by $\tau$ and canonical element denoted by $\xi$. We can define an inner involution on $\mathfrak{g}^{\mathbb{C}}$ by $\tau_{\xi}=\operatorname{Ad} \exp (\pi \xi)$, which induces a symmetric decomposition $\mathfrak{g}^{\mathbb{C}}=\mathfrak{k}^{\mathbb{C}} \oplus \mathfrak{m}^{\mathbb{C}}$, where

$$
\mathfrak{k}^{\mathbb{C}}=\sum_{i \text { even }} \mathfrak{g}_{i}, \quad \mathfrak{m}^{\mathbb{C}}=\sum_{i \text { odd }} \mathfrak{g}_{i} .
$$

Taking $K=(G)_{0}^{\tau_{\xi}}$, we get a symmetric space $N(F)=G / K$ with $H \subset K$. For example, $F=S O(2 n+1) / U(n)$ is a generalized flag manifold of height 2 and $N(F)=S^{2 n}$. Let $p$ : $F \rightarrow N(F)$ be the homogeneous projection. Following [6], we call this map the canonical twistor fibration associated to $F$. By Theorem 5.2 with $k=2$, we see that if $\psi: \mathbb{C} \rightarrow F$ is a primitive map of finite type, so is $p \circ \psi: \mathbb{C} \rightarrow N(F)$. Observe that in general, $N(F)$ is not a generalized flag manifold.

\section{References}

[1] M. Black, Harmonic Maps into Homogeneous Spaces, Pitman Research Notes in Mathematics Series, vol. 255, Longman Scientific \& Technical, Harlow; John Wiley \& Sons, New York, 1991.

[2] A. Borel and F. Hirzebruch, Characteristic classes and homogeneous spaces. I, Amer. J. Math. 80 (1958), no. 2, 458-538. 


\section{Primitive harmonic maps of finite type}

[3] F. E. Burstall, Harmonic tori in spheres and complex projective spaces, J. reine angew. Math. 469 (1995), 149-177.

[4] F. E. Burstall, D. Ferus, F. Pedit, and U. Pinkall, Harmonic tori in symmetric spaces and commuting Hamiltonian systems on loop algebras, Ann. of Math. (2) 138 (1993), no. 1, 173-212.

[5] F. E. Burstall and F. Pedit, Harmonic maps via Adler-Kostant-Symes theory, Harmonic Maps and Integrable Systems (A. P. Fordy and J. C. Wood, eds.), Aspects Math., E23, Vieweg, Braunschweig, 1994, pp. 221-272.

[6] F. E. Burstall and J. H. Rawnsley, Twistor Theory for Riemannian Symmetric Spaces. With Applications to Harmonic Maps of Riemann Surfaces, Lecture Notes in Mathematics, vol. 1424, Springer, Berlin, 1990.

[7] E. Calabi, Minimal immersions of surfaces in Euclidean spheres, J. Differential Geometry 1 (1967), 111-125.

[8] _ Quelques applications de l'analyse complexe aux surfaces d'aire minima, Topics in Complex Manifolds, Presses de Université de Montréal, Montréal, 1967, pp. 59-81.

[9] J. Eells and J. C. Wood, Harmonic maps from surfaces to complex projective spaces, Adv. in Math. 49 (1983), no. 3, 217-263, 79 (1957), 121-138.

[10] M. A. Guest, Harmonic Maps, Loop Groups, and Integrable Systems, London Mathematical Society Student Texts, vol. 38, Cambridge University Press, Cambridge, 1997.

[11] S. Helgason, Differential Geometry, Lie Groups, and Symmetric Spaces, Pure and Applied Mathematics, vol. 80, Academic Press, New York, 1978.

[12] J. E. Humphreys, Introduction to Lie Algebras and Representation Theory, Graduate Texts in Mathematics, vol. 9, Springer, New York, 1972.

[13] Y. Ohnita and S. Udagawa, Harmonic maps of finite type into generalized flag manifolds, and twistor fibrations, Differential Geometry and Integrable Systems (Tokyo, 2000), Contemp. Math., vol. 308, American Mathematical Society, Rhode Island, 2002, pp. 245-270.

[14] J. A. Wolf, The action of a real semisimple group on a complex flag manifold. I: Orbit structure and holomorphic arc components, Bull. Amer. Math. Soc. 75 (1969), 1121-1237.

Rui Pacheco: Departamento de Matemática, Universidade da Beira Interior, Rua Marquês d'Ávila e Bolama, 6201-001 Covilhã, Portugal

E-mail address: rpacheco@noe.ubi.pt 


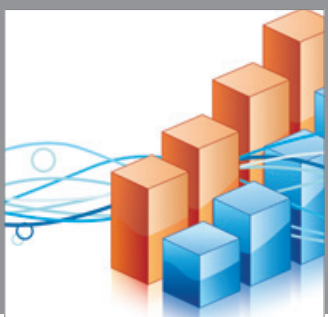

Advances in

Operations Research

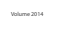

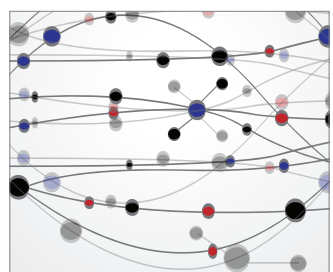

\section{The Scientific} World Journal
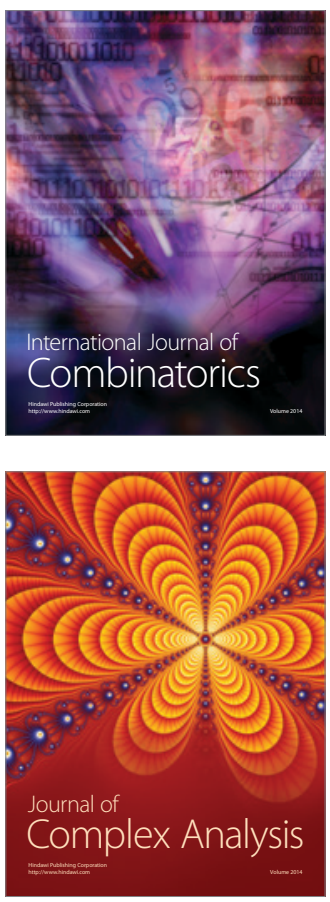

International Journal of

Mathematics and

Mathematical

Sciences
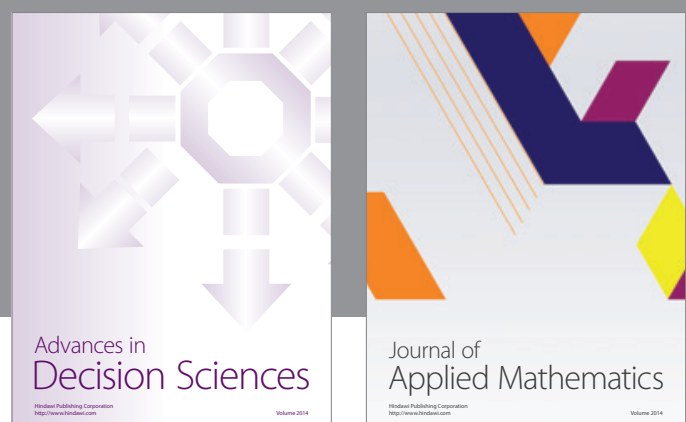

Journal of

Applied Mathematics
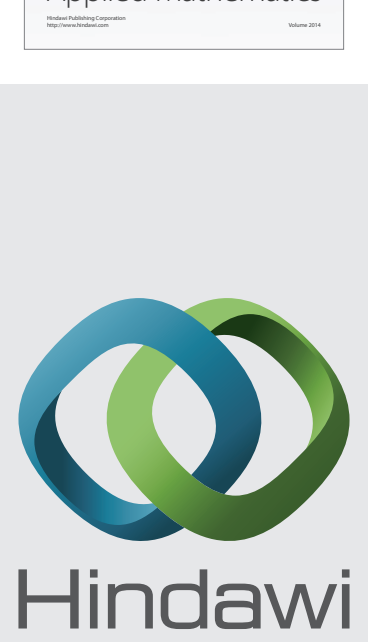

Submit your manuscripts at http://www.hindawi.com
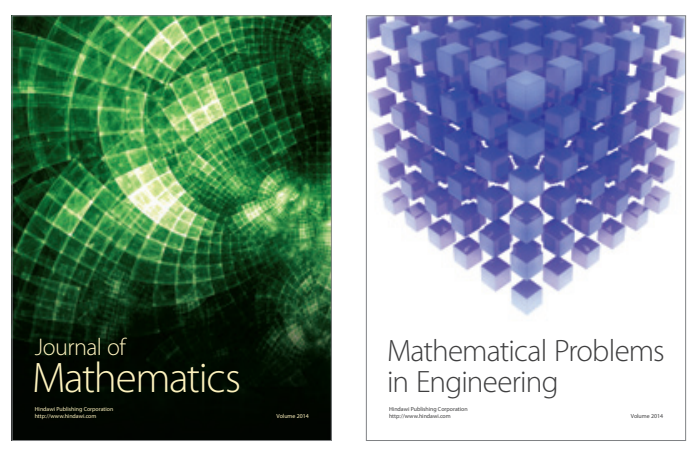

Mathematical Problems in Engineering
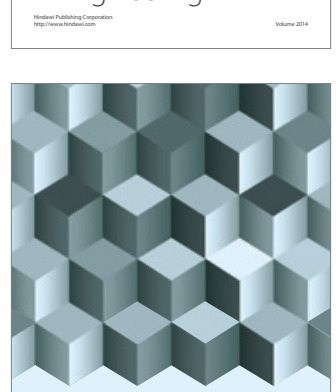

Journal of

Function Spaces
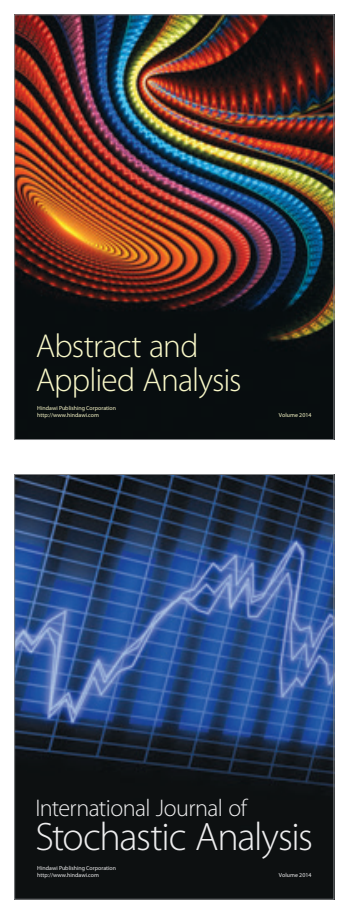

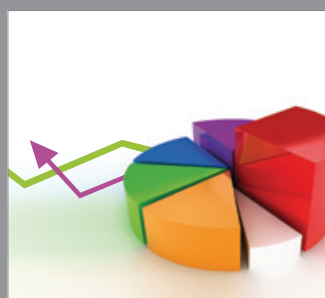

ournal of

Probability and Statistics

Promensencen
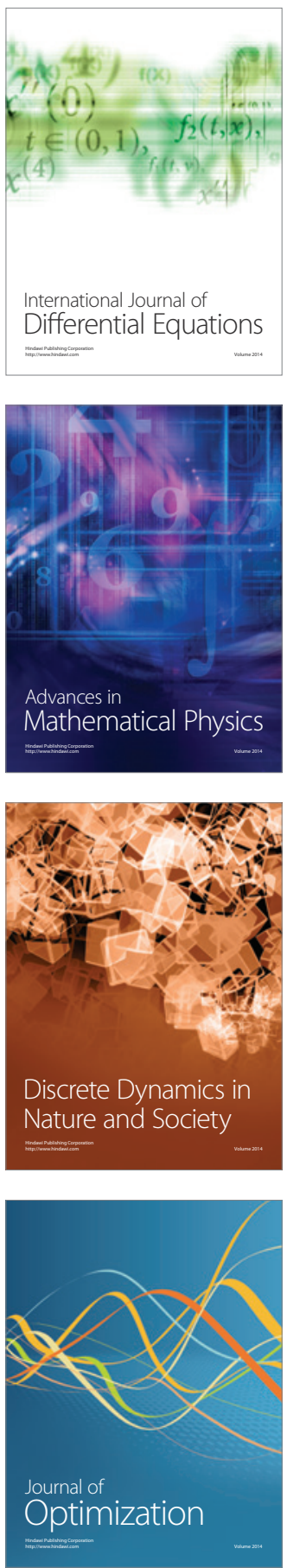